\title{
Competencia y precios en el mercado farmacéutico mexicano
}

\author{
Raúl E Molina-Salazar, Dr ${ }^{\left({ }^{\prime}\right)}$, Eloy González-Marín, candidato a doctor ${ }^{(2)}$, Carolina Carbajal-de Nova, M en C, ${ }^{(I)}$
}

Molina-Salazar RE, González-Marín E, Carbajal de Nova C. Competencia y precios en el mercado farmacéutico mexicano.

Salud Publica Mex 2008;50 supl 4:S496-S503.

\section{Resumen}

Las formas que asume la competencia en el mercado definen el nivel de precios. El mercado farmacéutico contiene submercados con diferente grado de competencia; por un lado existen productos innovadores con patente y, por el otro, genéricos con marca comercial o sin ella. Por lo general, los medicamentos innovadores con patente tienen precios monopólicos, pero a su vencimiento éstos bajan al enfrentar la competencia de alternativas terapéuticas. La marca permite conservar las rentas económicas del monopolio. En México los precios de los medicamentos en el mercado privado son elevados, de acuerdo con las estimaciones agregadas y para medicamentos específicos, lo cual refleja las limitaciones de la competencia en el mercado y el poder de la marca comercial. En el segmento público se obtienen precios competitivos con la estrategia de los medicamentos esenciales de la Organización Mundial de la Salud, con base en el listado de productos del Cuadro Básico.

Palabras clave: medicamentos esenciales; precios; patentes; México
Molina-Salazar RE, González-Marín E, Carbajal de Nova C. Competition and prices in the Mexican pharmaceutical market.

Salud Publica Mex 2008;50 suppl 4:S496-S503.

\section{Abstract}

The forms of market competition define prices. The pharmaceutical market contains submarkets with different levels of competition; on the one hand are the innovating products with patents, and on the other, generic products with or without trade names. Innovating medicines generally have monopolistic prices, but when the patents expire prices drop because of competition from therapeutic alternatives. The trade name makes it easier to maintain monopolistic prices. In Mexico, medicine prices in the private market are high -according to aggregated estimates and prices for specific medicines- which reflect the limitations of pharmaceutical market competition and the power of the trade name. The public segment enjoys competitive prices using the $\mathrm{WHO}$ strategy for essential medicines on the basis of the Essential List.

Key words: essential medicines, prices, patents

(I) Departamento de Economía, Universidad Autónoma Metropolitana, Iztapalapa, México, DF.

(2) Universidad Autónoma Metropolitana, Atzcapotzalco, México, DF.

Fecha de recibido: 9 de agosto de 2007 • Fecha de aceptado: 22 de julio de 2008 Solicitud de sobretiros: Dr. Raúl Molina. Departamento de Economía, Universidad Autónoma Metropolitana-Iztapalapa. Av. San Rafael Atlixco 186, col.Vicentina. 09340 Iztapalapa, México, DF. Correo electrónico:molinara_2005@yahoo.com.mx 
$\mathrm{L}$ a fabricación de medicamentos ha sido objeto de una gran transformación, y en su momento se llegó a postular que podría encontrarse un medicamento para casi todas las enfermedades: un antibiótico para infecciones, psicofármacos para la estabilidad mental, hormonas para el control de la fertilidad o de la artritis; y ahora, con la revolución de la biología molecular y de la ingeniería genética, parece que ese momento está muy cercano. A partir de la segunda guerra mundial, la industria farmacéutica inició un periodo de crecimiento con el lanzamiento de muchos medicamentos nuevos. Esta etapa dio origen al surgimiento de las grandes empresas transnacionales, las cuales modificaron la organización del mercado sobre la base de la investigación y el desarrollo de nuevos productos y el empleo de patentes, así como con el apoyo de las actividades de promoción y publicidad de marcas comerciales. ${ }^{1} \mathrm{El}$ mercado de medicamentos se organiza en submercados con diferente grado de competencia: a) un mercado de productos innovadores con patentes, $b$ ) un mercado de genéricos sin marca comercial al término de la patente, y c) un mercado de genéricos con marca comercial. Los medicamentos basados en nuevas moléculas con patente gozan de precios monopólicos, los cuales se erosionan con el surgimiento de alternativas terapéuticas o, al vencimiento de las patentes, con los medicamentos genéricos; de esta manera, se presenta una competencia de precios. ${ }^{2}$ La marca comercial constituye una estrategia de ventas para preservar las rentas económicas a que da lugar el monopolio.

En México, al igual que en otros países, se presenta un aumento en el gasto de medicamentos, lo cual genera una gran preocupación sobre cómo controlar este creciente gasto. La experiencia europea muestra que el control del gasto farmacéutico pide un enfoque integral en el cual el precio es un elemento más de dicha estrategia. ${ }^{3}$ Un rasgo especial del mercado mexicano es que el medicamento se financia principalmente del bolsillo de las personas, sobre todo los productos de patente, con marcas comerciales y precios lejanos a los de un mercado competitivo. En este trabajo se busca investigar sobre las formas de competencia y el nivel de los precios de los medicamentos en México.

\section{Competencia y precios: regulación en el mercado farmacéutico}

Los precios de los medicamentos han sido motivo de controversia entre todos los agentes que intervienen en la operación de los sistemas de salud, así como también para los pacientes que requieren del medicamento a fin de restaurar su salud, y esta inquietud en buena medida se debe a las fallas del mercado farmacéutico.
En el establecimiento de un sistema de control de precios, definir qué es un precio justo constituye todo un desafío. El precio de un producto en un mercado competitivo, sin barreras a la entrada, se acerca al concepto de precio justo, pero el mercado farmacéutico está muy lejos de operar en condiciones de competencia por las fallas que en él se presentan y que le son propias.

Las fallas del mercado farmacéutico asumen diferentes formas. ${ }^{4}$ Hay fallas intrínsecas en el mercado, como la asimetría de información que se presenta en el sentido de que el paciente tiene un conocimiento menor que el médico sobre las propiedades farmacoquímicas del medicamento; el paciente no tiene la formación adecuada para evaluar la seguridad y eficacia terapéutica del medicamento. Pero, además, la desigualdad en la información también se presenta entre el médico que recibe la información comercial y los laboratorios, como fue el caso de la talidomida de Ciba ${ }^{5}$, más recientemente, el de Vioxx de Merck. ${ }^{*}$ En ambos casos la búsqueda de ventas condujo a relegar las observaciones que sobre daños colaterales tenían estos medicamentos; tal ambición por vender provocó daños severos en los pacientes que usaron los productos mencionados.

Los monopolios artificiales que se generan con los derechos de propiedad intelectual a través de patentes y marcas registradas, reducen el grado de competencia efectiva en el mercado de medicamentos. ${ }^{6}$ El gasto en investigación y desarrollo de las empresas transnacionales farmacéuticas está muy por encima del gasto promedio industrial. Sin embargo, la industria ha venido afrontando una caída en la introducción de innovaciones reales, por lo que hace un uso extenso de innovaciones menores, cuyo propósito es la protección del mercado y el mantenimiento de precios elevados para conservar la rentabilidad de la industria, y no necesariamente ofrecer una mejor alternativa terapéutica.

A la par del gasto en investigación y desarrollo, está el gasto en promoción y publicidad de los medicamentos, pues una de las estrategias de marketing de la industria se finca en la diferenciación del medicamento. En países de ingresos elevados las instituciones de salud realizan la compra del medicamento, dado que se cuenta con una cobertura universal, con apoyo de un listado positivo o negativo de productos; pero en países de ingresos medianos y bajos, ante la ausencia de un financiamien-

\footnotetext{
* Merck tuvo que retirar el medicamento Vioxx cuando la Food and Drug Administration (FDA) de los Estados Unidos de América emitió una comunicación sobre los daños colaterales que podría ocasionar el medicamento, el 28 de septiembre de 2004. Disponible en: www. fda.gov/drug/ osteoarthritis/COX2/infopage/vioxx.default.
} 
to institucional para la adquisición de medicamentos, la inducción del médico se convierte en un elemento decisivo para la compra por parte de los pacientes.

La concentración del mercado de medicamentos es un asunto que también se presta a la discusión. Para México, el índice de Herfindal-Hirshman* arroja una cifra baja de concentración para la industria, pero en los submercados o clases terapéuticas se puede encontrar una situación diferente, como en el caso de los medicamentos para el sistema nervioso central, de los cuales sólo dos empresas controlan $47 \%$ del mercado total. ${ }^{7}$

En la industria farmacéutica es común encontrar precios diferentes para la misma sustancia activa, para el mismo producto y entre productos de marca y genéricos; estos patrones se repiten tanto en el plano local como en el internacional. Un estudio en Argentina proporciona información sobre las diferencias de precios para medicamentos con la misma marca, sustancia activa y forma farmacológica; ${ }^{8}$ información similar se encuentra para España. ${ }^{9}$ En otro estudio se comparan los precios de medicamentos con marca comercial y la misma presentación farmacológica en países europeos y en algunos países de ingresos bajos. ${ }^{10}$ Para el mercado de los antibióticos en México, se encuentra una gran diferencia de precios para productos con la misma sustancia activa y presentación farmacológica. ${ }^{11}$

Ante esta dispersión de precios, en países de ingresos elevados se tiene una intervención estatal directa en el mercado de medicamentos a través de regulaciones y controles, lo cual no ocurre en naciones de ingresos bajos. El control de precios en Europa abarca desde el costo de manufactura hasta precios de referencia con base nacional o internacional, así como límites a la tasa de ganancias y estudios de farmacoeconomía 12,13 (cuadro I). En España y Austria los precios se determinan a partir de los costos. En Alemania y Suecia se tienen precios de referencia para todos los productos. En Italia y Holanda los precios se fijan con base en el precio promedio de algunos países. En Francia los precios se fijan sobre la base de la efectividad de los medicamentos. En Inglaterra se tiene una regulación sobre la tasa de ganancia. ${ }^{14}$ En países como Australia, la introducción de nuevos medicamentos y su precio están definidos a partir de una evaluación económica sobre la costo-efectividad de los mismos. En Canadá se aplica un programa para el control de precios de pro-

\footnotetext{
* El índice Herfindal-Hirshman es una medida de concentración de la industria que puede tener un valor mínimo de 0 y un máximo de 10000 .
}

\section{Cuadro I \\ Estrategias DE CONTROL DE COSTOS EN EL SECTOR FARMACÉUTICO}

Estrategias de demanda

Operando sobre los pacientes

- Copago

- Ampliando la venta de mostrador (medicamentos sin receta)

- Programas de educación farmacéutica

Operando sobre los principales agentes (médicos y farmacéuticos)

- Sistemas de pago

- Médicos: capitación (pago per cápita)

- Médicos: salario

- Pago a farmacéuticos por porcentaje o por una cuota fija

- Presupuesto para el gasto farmacéutico

- Presupuesto fijo

- Presupuesto indicativo

- Políticas que estimulan la prescripción costo-efectiva

- Guías sobre práctica terapéutica

- Uso de estudios de costo efectividad

- Auditoría en la prescripción

- Genéricos: sustitución, promoción, etc.

Estrategias de oferto

Control de precios

Precios de referencia

Control de ganancias

Contribuciones industriales cuando se exceden los presupuestos

Control del número de productos

Techos en el gasto farmacéutico

Impuestos sobre el gasto de promoción

Desarrollo del mercado paralelo

Fuente: Referencia 3

ductos innovadores con patentes, a través del Patented Medicine Prices Review Board (PMPRB). ${ }^{15}$

Dentro de las estrategias de control de costos, una de las políticas de mayor efectividad ha sido la denominación genérica, pues a la vez que orienta la prescripción hacia el uso de los medicamentos esenciales, también restringe el papel de la marca comercial. ${ }^{16} \mathrm{La}$ Organización Mundial de la Salud (OMS) ha venido impulsando desde 1978 el Programa de Acción de Medicamentos Esenciales, ${ }_{1}^{17}$ es decir aquellos que logran satisfacer las necesidades de salud de la mayoría de la población a precios razonables, con base en su denominación genérica. La prescripción de genéricos ha aumentado en muchos países; se estima que representa más de $40 \%$ de las prescripciones totales en los Estados Unidos de 
América (EUA), Canadá y Alemania y hasta $80 \%$ en Inglaterra. En Australia se ha encontrado que las alternativas terapéuticas, al enfrentar la competencia de los nuevos medicamentos, inducen la caída de los precios de medicamentos basados en el omeprazol y de una terapia triple de antirretrovirales. ${ }^{18}$

En el segmento privado del mercado mexicano, que representa 85\% del valor total, la regulación contempla principalmente cuestiones relativas a la eficacia y seguridad de los medicamentos. En el ámbito económico se reduce al establecimiento de un precio máximo de venta al público para productos con patente vigente. Este precio es presentado por la propia empresa a la Secretaría de Economía, de acuerdo con lo establecido en el Artículo 31 de la Ley General de Salud. El precio máximo de venta al público se define a partir del precio promedio exfábrica del trimestre calendario inmediato anterior en los seis países con mayor participación en el mercado mundial. En el segmento de medicamentos con denominación genérica y de productos con marca comercial sin patente vigente, no existe regulación de precios. ${ }^{19}$ A pesar de la regulación, un estudio reciente de la Secretaría de Salud afirma que los precios en México son los más elevados de América Latina. ${ }^{20}$

Para el segmento público del mercado se emplean algunas de las estrategias de control que generan un gran ahorro a las instituciones del sector público. Por el lado de la demanda se han establecido políticas que estimulan la prescripción con base en su denominación genérica, la cual se acompaña de guías para la práctica terapéutica, de auditorías médicas y, en algunos casos, de estudios de costo-efectividad. Por el lado de la oferta se cuenta con el Cuadro Básico y Catálogo de Medicamentos, que limita el número de medicamentos. La compra de medicamentos en gran volumen se negocia con precios bajos. La Organización para la Cooperación y el Desarrollo Económico (OCDE) muestra que los precios del Instituto Mexicano del Seguro Social (IMSS) son $80 \%$ más baratos que los precios establecidos como máximos en los medicamentos de marca en el sector privado, ${ }^{19}$ y estudios anteriores ya habían mostrado que los precios negociados por el IMSS eran bajos. ${ }^{21}$

\section{Metodología}

Para el análisis de los precios, se estudian sus tendencias en el país así como la forma en que se ha realizado la comparación de los precios de algunos medicamentos mexicanos con los precios internacionales. Las comparaciones de precios internacionales al mayoreo con precios internos al detalle pueden producir información equívoca, dado que existen diferentes tasas impositivas y márgenes de intermediación que pueden oscurecer los resultados.

Para el análisis de precios al consumidor se toman los índices de precios tipo Laspeyres que elabora el Banco de México. El índice de precios tipo Laspeyres conlleva una canasta fija en la cual se subregistra el movimiento de los precios, pues los medicamentos nuevos con patente no tienen una estimación adecuada. De las cuentas nacionales se emplea el índice de precios implícitos.

Un estudio sobre la diferencia de precios calculada entre los principales mercados farmacéuticos cuestiona las estimaciones de la brecha entre los precios de los EUA y los de Canadá, donde los patrones de ponderación utilizados pueden distorsionar los resultados. Uno de ellos puede ser el ingreso monetario, para lo cual se pueden hacer algunos ajustes como propone Danzón; otro puede ser el patrón de consumo, pues el consumo de medicamentos en los EUA está muy influido por las empresas farmacéuticas. ${ }^{22}$

En la guía de MSH (Management Sciences for Health) se encuentra información de precios internacionales de los medicamentos en su denominación genérica. Los precios se estandarizan en dólares corrientes sobre la base de su concentración farmacológica.

Además, se hace un análisis de los medicamentos para hipertensión y antirretrovirales. Se comparan precios de productos de marcas líderes en el mercado, con sus referentes en su denominación genérica en el mercado internacional. Se consideran los precios de los productos al salir del laboratorio, sin los márgenes de operación al detalle y al mayoreo; la información de precios proviene de la empresa Autrey.

\section{Resultados}

En México, la razón de precios entre el índice de precios de la industria farmacéutica y el índice nacional de precios al consumidor durante las últimas dos décadas muestra que los precios de la industria farmacéutica crecen más que el índice nacional de precios al consumidor, como se desprende de la razón de precios (figura 1). Esta relación indica que, a partir de 1992, la inflación de la industria farmacéutica ha crecido en forma sostenida a una tasa mayor que la del resto de la economía (figura 1).

Una visión complementaria se ofrece con el índice de precios implícitos de la producción bruta farmacéutica, así como con el índice de volumen de la misma, provenientes ambos de las cuentas nacionales (figura 2). Este indicador muestra que en el mercado se ha registrado un fuerte crecimiento de precios en la última 


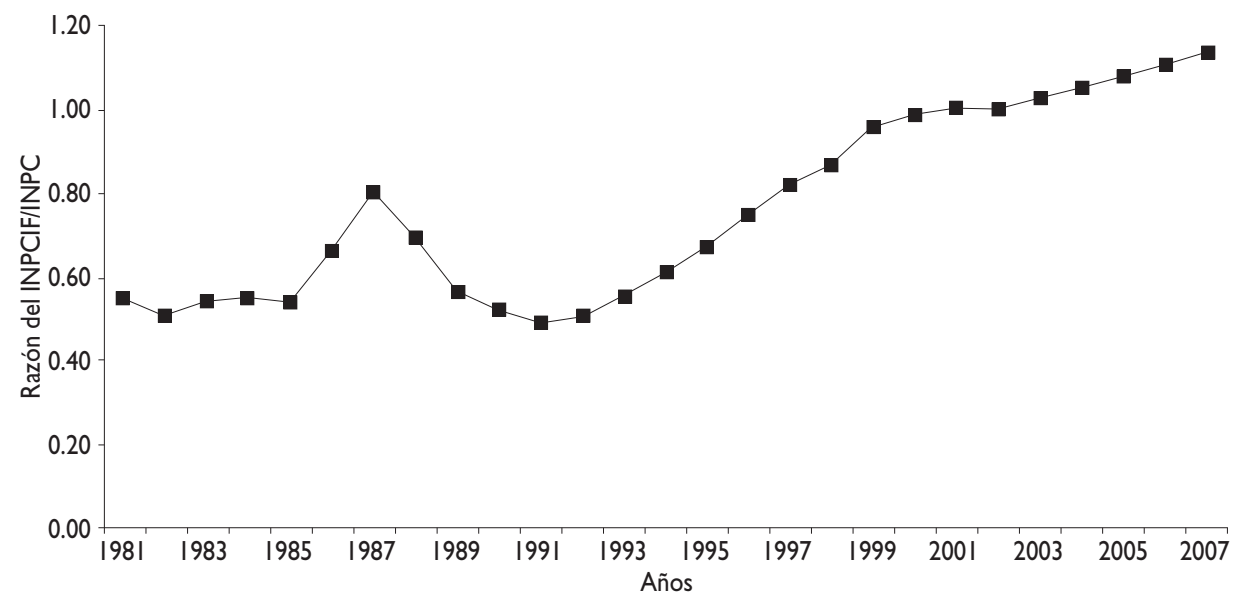

Fuente: Elaboración propia con datos del índice de precios del Banco de México: www.banxico.org.mx

Figura I. Razón del índice de precios al Consumidor de la industria farmacéutica (INPCIF) ResPecto aL índice de PRECIOS AL CONSUMIDOR (INPC). 198I-2007. MÉXICO. BASE 2002=100

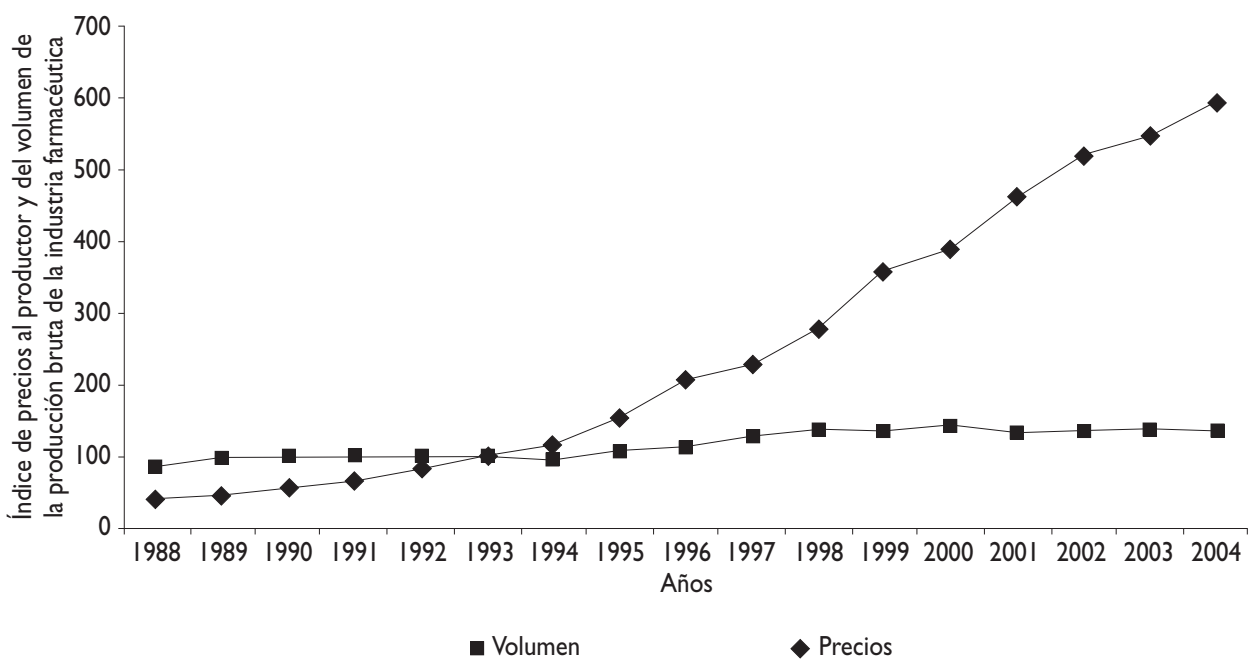

Fuente: Elaboración propia con base en La Industria Química en México 2007. Instituto Nacional de Geografía e Informática. México 2007

Figura 2. ÍNDICE de VOLUMEN de LA PRODUCCIÓN BRUTA E ÍNDICE DE PRECIOS AL PRODUCTOR DE LA INDUSTRIA FARMACÉUTICA. 1998-2004. BASE 1993=100

década, el cual no se ha acompañado de un crecimiento en unidades o la cantidad de los medicamentos.

En la figura 2 se puede observar que, a partir de 1988 y hasta 2004, el crecimiento del índice de precios de la industria farmacéutica presenta un fuerte aumento. En cambio, el índice de volumen presenta poco aumento durante el periodo de 1988 a 2004. Las estadísticas agregadas de la industria farmacéutica muestran un bajo crecimiento en las unidades físicas, contra un fuerte aumento en los precios. 
A continuación se analizan los precios de los medicamentos para el control de la hipertensión arterial (captopril) y de algunos antirretrovirales. En el caso del captopril ya se encuentran alternativas terapéuticas y en denominación genérica, aún cuando la empresa innovadora y líder de ventas mantiene la patente de algunas presentaciones. Los antirretrovirales que se analizan son productos con patente vigente en algunas presentaciones (didanosina, estaduvina, neviparina y lopinavir con ritonavir) y sin patente vigente (zidovudina).

El captopril es una sustancia que regula la presión arterial, y se patentó en 1977. El producto líder de ventas en su clase terapéutica se comercializa bajo el nombre de Capotena. Cuando se comparan los precios al mayoreo, se observa que, si bien en el plano internacional su precio ha registrado una caída frente a las presentaciones genéricas, lo opuesto sucede en el segmento del mercado privado en México, pues la marca comercial Capotena registra un alza en su precio (figura 3 ).

La comparación de los precios internacionales de algunos medicamentos antirretrovirales con los precios de compra que realiza el gobierno mexicano, muestra que la zidovudina presenta precios muy similares a los del mercado internacional; éste es un producto que se descubrió en 1964 en laboratorios de instituciones educativas y se patentó en 1986. Este fármaco no tiene patente vigente y existen alternativas en el mercado internacional, las cuales se reflejan en su precio (figura 4). El patrón de precios de los productos innovadores,

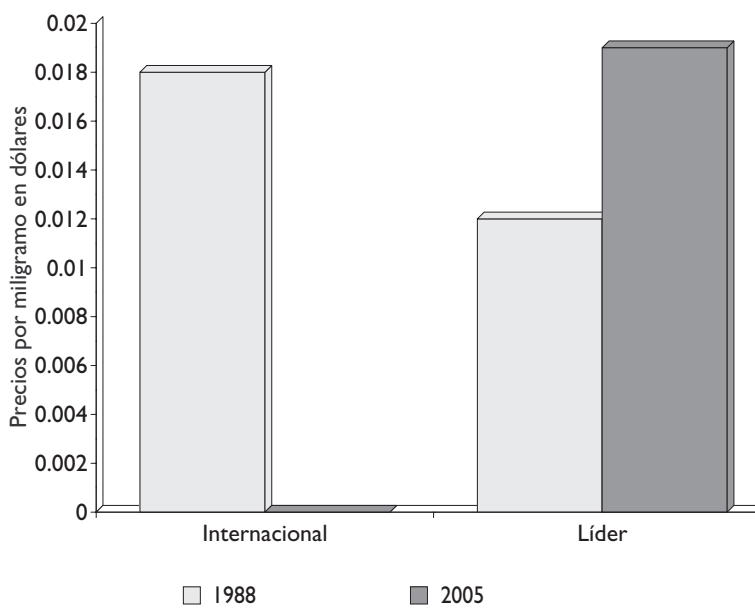

Elaborado por Erika Valerio con base en: Lista de precios de Casa Autrey e Internacional Drug Price Indicator Guide de MSH, en presentación de 25 mg, años 1998 y 2005

Figura 3. Comparación entre 1998 y 2005, de MediCAMENTOS PARA CONTROL de Hipertensión, PRECIOS POR MILIGRAMO (DÓLARES CORRIENTES)

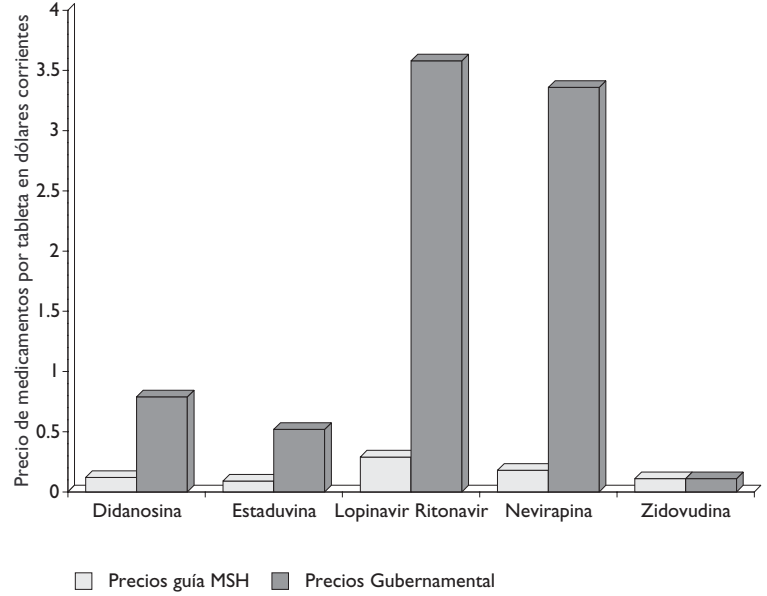

Elaboración propia con base en International Drug Price Indicator Guide de MSH y lista de precios de antirretroviales que publica CONASIDA en su sitio web www.conasida.gob.mx, 28 de febrero de 2007, precios por tableta en dólares corrientes

Figura 4. Precios de medicamentos antirRetrovirales POR TABLETA 2005 (DÓlARES CORRIENTES)

con patente vigente, presenta un patrón de precios diferente. La didanosina y la estaduvina son productos que provienen de la investigación de instituciones académicas públicas, las cuales otorgaron la licencia comercial en 1986 y 1991 respectivamente. A pesar de que en el mercado internacional se encuentran alternativas, el precio al que compra el gobierno es cuatro veces más elevado que el internacional. En el caso de la neviparina con la marca Viramune, cuya patente data de 1996, y del lopinavir con ritonavir, comercializado bajo la marca Kaletra, cuya patente data de 2004, el gobierno mexicano los compra a un precio más de 10 veces superior al de la alternativa internacional.

\section{Discusión}

La medición del precio de los medicamentos siempre ha sido un asunto de controversia tanto en México como en otras partes del mundo. Los precios de los medicamentos en el país han aumentado muy por encima del promedio en Latinoamérica, como se presenta en el estudio de la Secretaría de Salud. ${ }^{20}$ En el plano nacional, se encuentra que el índice de precios de los medicamentos aumenta más que el índice de precios al consumidor general, lo cual se refleja con claridad en la figura 1. El índice de precios implícitos muestra un aumento significativo en los precios de los medicamentos, al mismo tiempo que muy poco aumento de unidades, o sea que 
el crecimiento del valor del mercado se ha dado por el aumento de precios en los medicamentos.

La hipertensión es una enfermedad de elevada prevalencia en el país, de ahí la importancia de analizar los precios de los medicamentos para su tratamiento. El precio del captopril en dólares corrientes, en su formulación genérica, presenta un notable descenso a lo largo de una década en el mercado internacional. Sin embargo, el precio del captopril, con la marca comercial Capotena en México, presenta precios muy elevados en relación con su contraparte genérica. La marca comercial, en este caso, se constituye en un elemento que salvaguarda las rentas económicas de la patente en el mercado mexicano, a pesar del surgimiento de alternativas de tratamiento y genéricas en el mercado internacional.

El medicamento antirretroviral está diseñado para controlar los problemas generados por el VIH/SIDA, y puesto que esta enfermedad es una afección de reciente aparición, los medicamentos que se emplean en su tratamiento tienen patente. El gobierno mexicano ha venido ampliando la cobertura efectiva con el financiamiento público de antirretrovirales. La zidovudina, el primer fármaco que se empleó en el tratamiento del SIDA y cuya patente ya no está vigente, registra un precio bajo, similar al internacional. Pero en el caso de los otros productos estudiados en este trabajo, como son didanosina, estaduvina, lopinavir con ritonavir y nevirapina, el gobierno mexicano los compra a un precio muy elevado; el diferencial de precios se incrementa de manera significativa con los medicamentos más nuevos, cuyas alternativas terapéuticas son menores. El caso de Brasil ha sido muy comentado internacionalmente, pues la ampliación de la cobertura con terapia antirretroviral hasta el 100\% de las personas con VIH/SIDA se logró bajando los precios de los medicamentos; en algunos casos esta situación ha obligado a tramitar una licencia obligatoria de acuerdo con las salvaguardas que ofrece la Organización Mundial del Comercio, donde se privilegia el derecho a la salud sobre los derechos de propiedad intelectual. ${ }^{23}$

El gasto en medicamentos en proporción al gasto en salud se ha elevado en todo el planeta, y en los países de menores ingresos este porcentaje tiende a ser aún más elevado. La falta de un sistema de salud integrado en México es uno de los factores que eleva el gasto en medicamentos en proporción al gasto en salud, pues sólo en las instituciones del sector público se prescriben medicamentos genéricos de acuerdo con el Cuadro Básico y el Catálogo de Medicamentos, que contiene una lista de fármacos de probada eficacia terapéutica siguiendo los lineamientos de la OMS. Lo contrario ocurre en el segmento privado del mercado de medicamentos. El patrón de prescripción en el sector privado se orienta por los esquemas de promoción y ventas de las empresas, en los que se privilegia el uso de productos novedosos con marca comercial a precios elevados. Ante la ausencia de una evaluación terapéutica y económica de los medicamentos en México, se encuentra que el mercado farmacéutico registra un crecimiento en dólares constantes muy por arriba del crecimiento de la economía.

La introducción de nuevos medicamentos debería quedar restringida, junto con las consideraciones de eficacia y seguridad, a una evaluación económica de esos productos en la cual se delimiten con claridad las ventajas terapéuticas a través de estudios de costoefectividad, como ya se hace en Australia, Canadá y Nueva Zelanda. La evidencia empírica es concluyente acerca del menor precio de los medicamentos con denominación genérica, pues al eliminarse las restricciones a la competencia que representan las patentes y las marcas comerciales, el precio de los medicamentos se reduce.

Los precios a los que el IMSS adquiere los medicamentos, por su volumen de compra, se mueven a la par de los movimientos descendentes de precios de los medicamentos genéricos en el mundo. En la última década han aparecido en el mercado privado mexicano productos genéricos a precios reducidos, pero el poder de la marca comercial y de las patentes restringe la competencia en el mercado y se registran aún precios elevados en medicamentos, como se mostró antes. En este estudio se arroja luz sobre uno de los problemas del sistema de salud mexicano que impiden mejorar el acceso a los servicios de salud. Deben realizarse otros estudios sobre precios para diseñar una política de regulación y control de los mismos.

\section{Conclusiones}

- Los precios de los medicamentos en el mercado privado son elevados, de acuerdo con los índices de precios y en el caso de medicamentos específicos con marca comercial, lo cual es un reflejo de las barreras económicas que limitan la competencia en el mercado farmacéutico mexicano.

- En el segmento público del mercado se obtienen precios competitivos porque su utilización se rige por la estrategia de los medicamentos esenciales de la OMS, con base en una selección rigurosa de los productos incluidos en el Cuadro Básico y el Catálogo de Medicamentos, y con un alto volumen de compra.

- Se debe alentar la prescripción de medicamentos genéricos, como lo han venido haciendo otros países, tanto para lograr un uso más adecuado del medicamento como para estimular la compe- 
tencia en el mercado al eliminar la fuerte barrera económica que representa la marca comercial en los medicamentos.

- El gobierno de México tendrá que definir una política de evaluación económica de los medicamentos nuevos en la que se explicite cuál es el costo incremental de una ventaja terapéutica para definir su precio de incorporación al mercado.

\section{Referencias}

I. Sherer FM. La nueva estructura de la industria farmacéutica. En: Lobo F, Velásquez G. Los medicamentos ante las nuevas realidades económicas. Madrid: OMS-Universidad Carlos Tercero y Civitas, 1997: 207-227.

2. De Joncheere K, Rietveld AH, Huttin C. Experiences with generics. International Journal of Risk \& Safety in Medicine 2002; 15: 10I- 109. 3. Mossialos E, Le Grand J. Cost containment in the EU: an overview in health care and cost containment. En: Health care and cost containment in the European Union. Mossialos E, Le Grand J, eds. Cornwall:Ashgate, 1999: I-I54.

4. Stiglitz J. La economía del sector público. Barcelona:Antoni Bosh, 1997. 5. McBride WG. Thalidomide and congenital malformations. Lancet I96I; 2:1358.

6. Scherer F M. The pharmaceutical industry-Prices and progress. N Engl J Med 2004; 35I: 9:927-932

7. Durán L, Molina R, Jasso L. Financiamiento y precio de los medicamentos: El caso del IMSS. En: Martínez Salgado H, ed. Las múltiples facetas de la investigación en salud 4. México: IMSS, 2005.

8. Fundación ISALUD. El mercado de medicamentos en la Argentina. Buenos Aires: Secretaría de Industria Comercio y Minería, 1999. 9. Galdón C. Innovación y progreso en la industria farmacéutica. Madrid: Doce Calles, 1996.
10. Brudon P. ¿Medicamentos para todos en el año 2000? Las transnacionales farmacéuticas suizas frente al tercer mundo: el caso de México. México: Siglo XXI, 1987.

I I. Molina-Salazar RE, Rivas-Vilchis JF. Medicamentos, economía y salud. Aspectos económicos y utilización médico-social de los medicamentos. México:UAM-I Serie Texto y Contexto, 1992.

12. Mossialos E. El impacto sobre los medicamentos de la contención del gasto y de las reformas en la asistencia sanitaria. En: Lobo F,Velásquez G, eds. Los medicamentos ante las nuevas realidades económicas. Madrid: OMS-Universidad Carlos Tercero y Civitas, 1997;109-149.

13. Rietveld A, Haaijer-Ruskamp FM. Policy options for cost containment of pharmaceuticals. International Journal of Risk \& Safety in Medicines 2002;15:29-54.

14. Puig-Junoy J. Sistemas de regulación de los precios en el mercado farmacéutico. En: Puig-Junoy J.Análisis económico de la financiación pública de medicamentos. Barcelona:MASSON,2002: 17-39. 15. Gross D. Prescription drug prices in Canada. Washington, Issue Brief IB62 AARP Public Policy Institute, 2002.

16. De Joncheere K, Rietveld AH, Huttin C. Experiences with generics. International Journal of Risk \& Safety in Medicine 2002: 15: I0I-109.

17.World Health Organization. Resolution of the 3Ith Assembly, WHA3I.3I, 23th May 1978.

18. Henry D, Lexchin Jl. The pharmaceutical industry as a medicines provider. Lancet 2002:360:1590-1595

19. Moïs P, Docteur E. Pharmaceutical pricing and reimbursement policies in Mexico. Paris, OECD DELSA/HEAP, Health Working Papers No 25, 2007. 20. Secretaría de Salud. Hacia una política farmacéutica integral para México. México: SSA, 2005

21. Molina-Salazar RE. Estructura de mercado y precios. El caso de los medicamentos esenciales. En: La industria y las finanzas en el México actual. México: UAM-I, Serie de Investigación I4, 1995:69-82.

22. Danzon PM, Furukawa M. Prices and availability of pharmaceuticals : evidence from nine countries. Health Aff (Millwood) 2003:W3-52I -W3526.

23. Friedman $M$, den Besten $H$,Attaran A . Out-licensing: a practical approach for improvement of access to medicines in poor countries. Lancet 2003; 36I: 34I-44. 